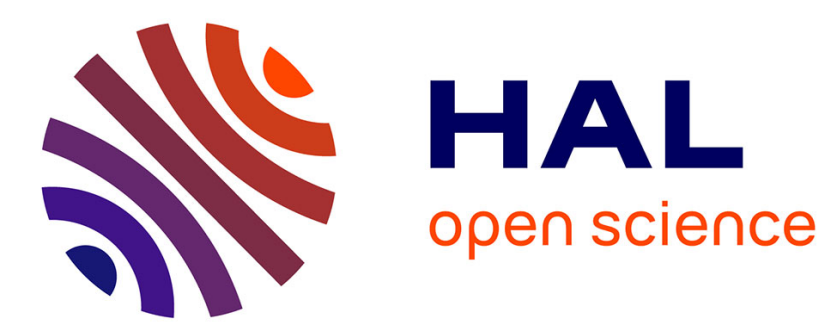

\title{
Trait-dependency of trophic interactions in zooplankton food webs
}

Florian Vincent, Andrea Bertolo, Gérard Lacroix, M.A. Mouchet, Eric Edeline

\section{To cite this version:}

Florian Vincent, Andrea Bertolo, Gérard Lacroix, M.A. Mouchet, Eric Edeline. Trait-dependency of trophic interactions in zooplankton food webs. Oikos, 2020, 129 (6), pp.891-902. 10.1111/oik.06783 . mnhn-02920029

\section{HAL Id: mnhn-02920029}

\section{https://hal-mnhn.archives-ouvertes.fr/mnhn-02920029}

Submitted on 24 Aug 2020

HAL is a multi-disciplinary open access archive for the deposit and dissemination of scientific research documents, whether they are published or not. The documents may come from teaching and research institutions in France or abroad, or from public or private research centers.
L'archive ouverte pluridisciplinaire $\mathbf{H A L}$, est destinée au dépôt et à la diffusion de documents scientifiques de niveau recherche, publiés ou non, émanant des établissements d'enseignement et de recherche français ou étrangers, des laboratoires publics ou privés. 


\section{Trait-dependency of trophic interactions in zooplankton food webs}

2

Florian Vincent ${ }^{1}$, Andrea Bertolo ${ }^{2}$, Gérard Lacroix ${ }^{1,3}$, Maud Mouchet ${ }^{4}$, Eric Edeline ${ }^{1,5 *}$

4

1: Sorbonne Université, Université Paris Diderot, UPEC, CNRS, INRA, IRD, Institut d'Ecologie et des

6 Sciences de l'Environnement de Paris (iEES-Paris), Paris, France. Email: fvincent.world@gmail.com

2: Département de sciences de l’environnement, Université du Québec à Trois-Rivières, 3351 Boul. des

8 Forges, CP 500, Trois-Rivières, Québec G9A 5H7 Canada. Email: andrea.bertolo@uqtr.ca, ORCID iD: https://orcid.org/0000-0002-6018-816X

103 : Ecole Normale Supérieure, PSL Research University, CNRS, Centre de recherche en écologie expérimentale et prédictive (CEREEP-Ecotron IleDeFrance), UMS 3194, 11 chemin de Busseau,

1277140 Saint-Pierre-lès-Nemours, France. Email: gerard.lacroix@upmc.fr

4: Maud Mouchet, UMR MNHN-CNRS-SU 7204 CESCO, 43 rue Buffon, CP 135, 75005 Paris. Email:

14 maud.mouchet@mnhn.fr

5: INRAE, Ecology and Ecosystem Health, 65 rue de Saint-Brieuc, 35042 Rennes Cedex, France.

16 Email: eric.edeline@inra.fr

18 * Corresponding author:

Email: eric.edeline@inra.fr,

20 ORCID iD: https://orcid.org/0000-0003-4963-4453 


\section{Declarations}

Acknowledgements - AB and GL warmly thank Françoise Lescher-Moutoué for her contribution in

24 conceiving the experimental design and for her contribution to the experiment.

Funding - FV was funded by a grant from Sorbonne Université (CARESE-SU J15R323). GL and FV

26 were supported by the International Program For Scientific Cooperation (project PICS06763) of the French National Center of Scientific Research. AB was funded by a NSERC Discovery grant (RGPIN-

28 2017-05451). EE was supported by a research grant from Rennes Métropole (AIS program - project number 18C0356).

30 Author contributions - All authors designed the study, AB and GL performed the experiment and produced the data, FV and EE analysed the data, all authors wrote the paper.

32 Conflicts of interest - The authors declare no conflict of interest.

Permit(s) - Fish manipulations were performed under the control of authorities and in compliance with 34 applicable laws. 


\section{Introduction}

2 Trophic interactions determine population dynamics, evolutionary trajectories and the stocks and fluxes of energy in ecosystems (Polis and Winemiller 1996). Therefore, uncovering general rules that shape

4 trophic interactions represents a central objective of ecological research. To reach this goal, researchers classically either use a priori-built models whose predictions are compared to observed food webs, or

6 infer general rules a posteriori through mechanistic or statistical models fitted to observed networks (Rohr et al. 2017). Both approaches require high-quality, detailed food-web data built from direct

8 observations. Such data are scarce and costly to acquire. A third, cost-effective but indirect approach to inferring trophic interactions is to experimentally measure community response to manipulating

10 presence-absence of particular predators in the food web (Hulot et al. 2014).

12 Experimental manipulations of predator-prey systems have a long history in ecological research and, in particular, have proven powerful to reveal whether and how trophic interactions are predictable from

14 single-trait phenotypes (Bolker et al. 2003, Werner and Peacor 2003, Schmitz 2008). Such studies have, for instance, confirmed the major role played by body size in structuring trophic interactions

16 (Emmerson and Raffaelli 2004), a role that was already envisioned by Elton (1927) and robustly explored since then (Cohen et al. 1993, Woodward et al. 2005, Brose et al. 2006). Recently, such

18 manipulative experiments have demonstrated that predator hunting mode interacts with prey antipredator behaviour in controlling predator-prey interactions and, from there, in determining both

20 the strength of trophic cascades (Schmitz et al. 2004, Schmitz 2008) as well as the architecture of whole food webs (Lazzaro et al. 2009). 
Besides body size, hunting mode and antipredator behaviour, other phenotypic traits may be involved

24 in controlling the occurrence and strength of trophic links (e.g. palatability, chemical defences or mimicry). However, these other traits are often difficult to identify or measure, and are poorly reported

26 in the literature. To tackle this complexity issue, phylogeny may be used as a "holistic trait" encapsulating the complex phenotypes that determine trophic niches. Accordingly, several studies

28 suggest that phylogeny (using taxonomy as a proxy) has a higher power than body size in predicting trophic links in observed terrestrial and aquatic food webs (Cattin et al. 2004, Naisbit et al. 2012).

30 Recently, some authors have further suggested that even a coarse phylogenetic resolution might still retain enough information to efficiently predict trophic links (Gauzens et al. 2013).

To our knowledge, however, no study has quantified the respective contributions of body size, 34 antipredator behaviour, and phylogeny at different resolutions in driving the presence and strength of trophic interactions in complex food webs. To contribute bridging this knowledge gap, we measured

36 the numerical response of a zooplankton community to the experimental manipulation of their fish predators in lake enclosures. We expected fish predation to have both direct effects through fish38 zooplankton trophic interactions, and indirect effects through reorganizations of zooplanktonzooplankton and zooplankton-phytoplankton trophic interactions. To investigate the role of

40 zooplankton traits in driving both these direct and indirect effects, we grouped zooplankters based on their body size, antipredator behaviour or on both their body size and antipredator behaviour, and we

42 made qualitative predictions on whether and how each zooplankton group should numerically respond to fish. Specifically, we predicted fish to deplete large-bodied zooplankton, and to favour increased 44 abundances of small-bodied zooplankton due to relaxed predation and competition from large-bodied zooplankton. We further predicted that, at a constant total fish biomass, smaller-bodied fish should have 46 a higher cumulated attack rate on large-bodied zooplankton. Based on antipredator behaviour, we 
predicted (i) "fleeing" zooplankton strategists to efficiently escape predation from cruising-foraging

48 fish but not from visual-feeding fish, (ii) "hiding" zooplankton strategists to efficiently escape predation from both type of fish, and (iii) "no escape" zooplankton strategists to be equally depleted by

50 both type of fish. Adding the effects of body size on top of the effects of antipredator behaviour changed (i) the predicted response of small-bodied, "no escape" zooplankton strategists to fish from

52 negative to positive, and (ii) the predicted response of "hide" zooplankton strategists from nil to positive because they feed on small-bodied, "no escape" zooplankton. We formulate more detailed and

54 referenced predictions in the Methods section.

56 We compared these qualitative predictions with observed effect sizes and, additionally, we adopted a model selection procedure that quantified the relative power of size- and behaviour-based zooplankton

58 groups to predict zooplankton response to fish manipulation. We further grouped zooplankters based on phylogeny at a low, medium or high resolution, and included these phylogeny-based groups into the

60 model selection procedure. In doing so, we combined phylogeny-based grouping with ontogeny-based grouping among Copepods (i.e., nauplii vs. copepodites and adults), as is usual when describing

62 zooplankton communities. We show that body size-based zooplankton grouping outperformed behaviour-based zooplankton grouping in predicting zooplankton numerical response to fish, but

64 performed less well than a grouping structure combining both zooplankton body size and behaviour. A phylogeny-based zooplankton grouping structure provided the overall best and most parsimonious fit to

66 the data, and was therefore most powerful at predicting zooplankton numerical response to fish predators, but only at a high to medium phylogenetic resolution and when ontogeny was also accounted 68 for. 


\section{Experimental setup}

Nine enclosures made of translucent polyethylene were installed on a floating pontoon on Lake Créteil,

74 a 42-ha shallow lake of $4 \mathrm{~m}$ mean depth (max depth $6 \mathrm{~m}$ ). Whereas they dit not include lake sediments, our enclosures were $1.5 \mathrm{~m} \times 1.5 \mathrm{~m} \times 4.5 \mathrm{~m}$ deep $\left(9.5 \mathrm{~m}^{3}\right)$ and, hence, realistically reproduced lake

76 vertical heterogeneity. Enclosures were filled with lake water pumped from a 1.5-meter depth from June $29^{\text {th }}$ to July $3^{\text {rd }} 1995$. After filling, enclosures were enriched with living zooplankton sampled from

78 the pelagic area of the lake. In order to avoid nutrient limitation of phytoplankton, inorganic nutrients were added twice a week with a liquid mixture of potassium phosphate $\left(\mathrm{KH}_{2} \mathrm{PO}_{4}\right)$ and ammonium

80 nitrate $\left(\mathrm{NH}_{4} \mathrm{NO}_{3}\right)$ with a $\mathrm{N}: \mathrm{P}$ ratio of 20:1 by weight, for a load of phosphorus of $3.16 \mu \mathrm{L} \mathrm{L}^{-1} \mathrm{~d}^{-1}$. At the start of the experiment (July $13^{\text {th }}$ ), the zooplankton biomass was $>200 \mu \mathrm{g}$ dry weight $\mathrm{L}^{-1}$. Additional

82 details on the experimental setup can be found in Bertolo et al. (1999b) and in Bertolo et al. (2000).

84 On July $13^{\text {th }}$, three different triplicate treatments were applied randomly to the enclosures: (i) “Control": fishless; (ii) "Perch": 18 planktivorous perch (aged 0+), measuring $5.97 \mathrm{~cm} \pm 0.55 \mathrm{~cm}$ (fork

86 length) and weighting $2.45 \pm 0.76 \mathrm{~g}$ (fresh weight); (iii) "Roach": six roach aged of at least a year $(1+)$, measuring on average $8.13 \pm 0.37 \mathrm{~cm}$ and weighting on average $7.50 \pm 0.50 \mathrm{~g}$. The different numbers

88 of roach and perch per enclosure were used to standardize fish biomass at 20 g.m ${ }^{-2}$, which can be considered as a moderate stocking rate (Williams and Moss 2003). Hence, in our design fish identity is

90 confounded with fish density, body size and hunting mode (see below), and we could thus not identify which fish traits would actually determine different zooplankton response when comparing perch to 
92 roach. For this reason, our study is based on exploring zooplankton rather than fish traits and how these influence zooplankton response to fish.

94

\section{Sampling}

96 In order to alleviate constraints inherent to mesocosms (higher fish biomass than in natural systems, limited movements of zooplankters, and severe prey depletion), we limited the duration of the

98 experiments to 20 days. Water was sampled at every $30-\mathrm{cm}$ depth in each enclosure using a $2-\mathrm{L}$ Friedinger bottle on three dates (July $18^{\text {th }}$ and $25^{\text {th }}$, August $1^{\text {st }} 1995$ ). All samples from the same

100 enclosure were pooled ( $24 \mathrm{~L}$ in total). Zooplankton of the pooled sample was filtered through a $50 \mu \mathrm{m}$ sieve and fixed in $4 \%$ formalin. One to two litres of $50 \mu \mathrm{m}$-sieved water (depending on filter clogging)

102 were filtered on a GF/C glass fiber filter (nominal cut-off: $1.2 \mu \mathrm{m}$ ) for estimation of chlorophyll $a$ content. Algal cells were strongly dominated by small algae $(<25 \mu \mathrm{m})$ mainly belonging to

104 Chlorophyceae, Cryptophyceae and diatoms (Bertolo et al. 1999b, 2000), and a negligible fraction was retained on the $50 \mu \mathrm{m}$ sieve. Chlorophyll $a$ was measured spectrophotometrically $\left(\mu \mathrm{g} \mathrm{L}^{-1}\right)$ after 106 grinding the samples in 90\% acetone and centrifugation (AFNOR 1990).

108 Before being analysed under a stereomicroscope, each zooplankton sample was subsampled and diluted so as to standardize abundances. Zooplankton counts from diluted subsamples were then transformed to

110 zooplankton concentration (mean count $\mathrm{L}^{-1}$ ) in each enclosure. Zooplankton individuals were identified at the species level when only one species was present and at the genus level when two congeneric

112 species co-occurred. Copepods were present in two development stages, specifically as nauplii vs. copepodites and adults (Reid and Williamson 2010). Copepod nauplii were segregated into nauplii of

114 Calanoids and nauplii of Cyclopoids. Cladocera, and copepodites of Copepods were measured for body length (in mm, 40 individuals per zooplankton category and per sample). 


\section{Zooplankton taxa}

118 We identified five herbivorous cladoceran genera: Ceriodaphnia [C. pulchella Sars 1862 and C. quadrangula (O.F. Müller 1785)], Bosmina [B. longirostris (O.F. Müller 1785)], Diaphanosoma [D.

120 brachyurum (Liévin 1848)] and Daphnia (D. longispina complex), listed according to their relative abundance. The omnivorous copepods were represented by the Calanoid species Eudiaptomus gracilis

122 (Sars 1863) and the Cyclopoid species Acanthocyclops robustus (Sars 1863), Thermocyclops crassus (Fischer 1853), and Th. oithonoides (Sars 1863). The herbivorous rotifers were dominated by

124 Polyarthra sp. and Hexarthra mira (Hudson 1871), while the predatory genus Asplanchna (A. girodi De Guerne, 1888, A. priodonta Gosse, 1850) represented the carnivorous rotifers. Other zooplankton

126 taxa, such as undetermined Chydorids, the calanoid Copepod Eurytemora velox (Lilljeborg 1853), and the rotifers Keratella quadrata (O.F. Müller 1786), K. cochlearis (Gosse 1851), and Lecane spp., were

128 only found very occasionally and were not taken into account in the subsequent analyses.

\section{Assigning zooplankton taxa to trophic groups from prior knowledge}

We assumed that the assembly of zooplankton trophic groups (and associated trophic interactions with

132 fish and other zooplankters) was controlled by zooplankton (i) body size only, (ii) antipredator behaviour only, (iii) body size and behaviour combined, and (iv) phylogeny (using taxonomy as a

134 proxy) at different resolutions and combined with ontogeny for Copepod taxa. Below, we present the criteria used to assign zooplankton taxa into trophic groups and predict their associated numerical 136 response to perch and roach (summarized in Table 1).

138 Body size-based zooplankton trophic groups 
During summer, zooplankton in Lake Créteil include few large-bodied organisms and thus have a

140 relatively narrow body-size range. They may be separated among small-bodied organisms (50-200 $\mu \mathrm{m})$, which include mainly herbivores (Rotifers and nauplii of Copepods), and medium to large-bodied

142 organisms $(200-2000 \mu \mathrm{m}$, among which $8.7 \%$ were $>1000 \mu \mathrm{m})$, which include both herbivorous or omnivorous/carnivorous microcrustaceans and carnivorous Rotifera (Bertolo et al. 1999a). In order to

144 take into account other realistic ways of classifying zooplankton, we split it into three size classes: 50$200 \mu \mathrm{m}$ (Rotifers and nauplii of Copepods), 200-600 $\mu \mathrm{m}$ (Bosmina, Ceriodaphnia and Thermocyclops)

146 and 600-2000 $\mu \mathrm{m}$ organisms (Asplanchna, Daphnia, Diaphanosoma, Eudiaptomus, Acanthocyclops). When fitting models to zooplankton count data (see below), however, this three size-class grouping 148 structure did not perform better than the two size-class grouping structure (LR test: Chi-square = 2.5382, $\mathrm{Df}=3, \mathrm{p}=0.4684$ ), and was penalized in terms of Akaike's information criterion (AIC, 2674.1

150 vs. 2670.6). We therefore retained the two-size-classes grouping structure for further analyses.

152 Fish preferentially consume large-bodied zooplankters, while small-bodied zooplankters are both dominated competitors and prey for larger zooplankton (Brooks and Dodson 1965). Hence, we

154 predicted that small-bodied zooplankton abundance should increase in the presence of fish (Table 1, Body size). Moreover, based on perch and roach differences in body size, we predicted perch to impose

156 a stronger predation pressure than roach and thus, in turn, to have a larger positive effect on smallbodied zooplankton (Table 1, Body size). This prediction emerges from general body-mass scaling

158 rules, where average attack rate for freshwater vertebrate ectotherms equals $8.2 \cdot 10^{-6} \mathrm{~m}^{0.31}$ (where $m$ is individual body mass, Rall et al. 2012). Using this relationship, cumulated attack rate on 160 zooplankton from 18 perch weighing $2.45 \mathrm{~g}$ is predicted to be twice as large than that from six roach weighing $7.50 \mathrm{~g}$. Finally, we predicted this higher cumulated attack rate from perch to result in a 
162 stronger trophic cascade (i.e. increase in phytoplankton abundance) than in the presence of roach (Table 1, Body size).

164

\section{Behaviour-based zooplankton trophic groups}

166 Possible behavioural responses of zooplankters facing predation from fish or from other invertebrates may be either (i) "fleeing" reaction, (ii) hiding in a spatial refuge or (iii) no escape. Whereas these

168 behaviour-based zooplankton trophic groups are not mutually exclusive, we assigned each zooplankton organism to only one group based on prior literature knowledge about their predominant behaviour.

Specifically, nauplii of both Calanoid and Cyclopoid copepods have limited escape capabilities, while

172 copepodite stages of are often equally categorized as "flee" strategists due to their jump-swimming behaviour. However, Calanoids occupy the upper water layers at both the nauplius and copepodite

174 stages (Angeli et al. 1995, Lacroix and Lescher-Moutoué 1995, Lieschke and Closs 1999, Bertolo et al. 1999a, Titelman and Fiksen 2004, Doulka and Kehayias 2011), and thus can not avoid encounters with

176 fish. Hence, we categorized Calanoids as "no escape" strategists at the nauplius stage and as "flee" strategists at the copepodite stage. In contrast, Cyclopoids prefer the deep layers of the water column in

178 Lake Créteil (Lacroix and Lescher-Moutoué 1995) and may even be regularly found in near-bottom habitats at both the nauplius and copepodite stages (Papińska and Prejs 1979, Papińska 1981). Hence,

180 Cyclopoids may efficiently hide from fish predators by preferentially occupying deep water layers. We considered hiding as a more efficient antipredator strategy than fleeing, and we categorized Cyclopoids

182 as "hide" strategists at both the nauplius and copepodite stages.

184 We also categorized as "flee" strategists Diaphanosoma spp., a jump-swimmer rapidly alternating sinking with powerful strokes of antennae (Williamson 1983, Chang and Hanazato 2003), but also 
186 Bosmina spp., which use a "dead-man response" (in addition to having a hard carapace) to deter predation from copepods (Kerfoot 1978, Williamson 1983, Sakamoto and Hanazato 2008). Although 188 jumping and a dead-man response are sharply different behaviours, we grouped them in a common "flee" category because their common purpose is to reduce mortality from unavoidable predator

190 encounters. This choice was further justified a posteriori by fitting statistical models to zooplankton count data. A model considering Bosmina separately as a "passive escape" strategist did not fit the data

192 better than a model grouping Bosmina together with Diaphanosoma and copepodites of Calanoids (LR test: Chi-square $=3.8594, \mathrm{Df}=3, \mathrm{p}=0.2771)$, and was penalized in terms of AIC (2688.7 vs. 2686.6).

Finally, Daphnids have a reduced ability to escape by jumping. Additionally, in Lake Créteil both 196 Ceriodaphnia spp. and Daphnia spp. preferentially use the upper layers of the water column (Lacroix and Lescher-Moutoué 1995), and thus are highly susceptible to predation by planktivorous fish. We 198 thus categorized Daphnids as "no escape" strategists, together with Rotifers.

200 Perch and roach also have contrasted hunting modes. Planktivorous perch are typical visual feeders (Lacroix et al. 1996) able to feed on zooplankton only when light intensity is relatively high (Nurminen 202 et al. 2010, Kestemont et al. 2015). They are stimulated by conspicuous prey, and prey movement can trigger an attack (Peterka and Matěna 2009). Perch are stop-and-burst predators that can repeatedly 204 strike, particularly so when feeding on evasive copepods (Peterka and Matěna 2011). Hence, we predicted perch to efficiently deplete both "no escape" and "fleeing" zooplankton strategists, but to be 206 less efficient on "hide" strategists (Table 1, Behaviour).

208 In contrast with perch, roach are cruising foragers that use continuous and rapid swimming, punctuated by slowdowns (Peterka and Matěna 2011, Helenius et al. 2015). They are able to switch between 
210 particulate feeding and gulping, depending on zooplankton body size, abundance and light conditions (Van Den Berg et al. 1993, Helenius et al. 2015). Roach foraging efficiency is almost independent of

212 the light intensity (Diehl 1988, Nurminen et al. 2010), indicating that they only optionally rely on visual cues to locate their prey. Moreover, even when light intensity is relatively high, roach are poorly

214 capable to prey on "fleeing" zooplankton strategists like copepods (Lacroix et al. 1996, Peterka and Matěna 2009, 2011). Hence, we expected roach in our experiment to deplete "no escape" zooplankters,

216 but not "fleeing" or "hide" strategists (Table 1, Behaviour).

\section{Mixture of size- and behaviour-based zooplankton trophic groups}

Body size should be important to predict zooplankton-fish trophic links only for zooplankters that are

220 unable to behaviourally avoid encounters with fish. Hence, we separated "no escape" zooplankton strategists into "large-bodied" and "small-bodied" zooplankton trophic groups. "Fleeing" strategists

222 were similar in body sizes and were thus not partitioned into different body size-based zooplankton trophic groups. Finally, "hide" strategists, by efficiently avoiding predation from both perch and roach,

224 were free from any size-dependency in their response to fish and were also not partitioned into different body size-based zooplankton trophic groups. Because they are omnivorous, however, we predicted

226 "hide" strategists to increase in parallel with the increasing abundance of "small, no escape" strategists on which they feed. The predictions resulting from this zooplankton grouping structure are summarized 228 in Table 1 (Body size and behaviour).

\section{Phylogeny-based zooplankton trophic groups}

We identified 12 genera or species and two development stages for copepods (see above), yielding a

232 total of 14 trophic groups. Reaching this taxonomic resolution in zooplankton counts, however, is a time-consuming task which is relieved if taxa may be lumped. It is therefore important to quantify the 
234 effect of taxonomic lumping on information loss in predicting food-web structure (Gauzens et al. 2013). Accordingly, we varied zooplankton lumping in our dataset from low to high to reach a (i) low-

236 level lumping (12 trophic groups: Ceriodaphnia, Bosmina, Diaphanosoma, Daphnia, Eudiaptomus, Acanthocyclops, Thermocyclops, nauplii of Calanoids, nauplii of Cyclopoids, Polyarthra, Hexarthra,

238 Asplanchna), (ii) medium-level lumping (10 trophic groups: Daphnidae, Bosminidae, Sididae, Diaptomidae, Cyclopidae, nauplii of copepods, Hexarthridae, Synchaetidae, Asplanchnidae) and (iii)

240 high-level lumping (4 trophic groups: Cladocera, copepodite of copepods, nauplii of copepods, and Rotifera).

\section{Data analysis}

\section{Zooplankton counts}

Our analysis of zooplankton count data aimed at (i) quantifying the numerical response of the different

246 zooplankton trophic groups to the fish treatments, (ii) identifying which zooplankton grouping structure provided the best and most parsimonious fit to the data, and (iii) quantifying, for each zooplankton

248 grouping structure, the importance of considering perch and roach as having different effects on zooplankton numbers. We based our analysis on comparing the fit to zooplankton count data of the

250 resulting 12 different generalized linear mixed models (Table 2), which corresponded to the six zooplankton grouping structures described above times two different fish effects: fish absent vs. fish

252 present or fish absent vs. perch present vs. roach present. We provide below more details on the analyses.

Zooplankton counts $C$ included a high proportion $(25 \%)$ of zeros, and positive counts were highly

256 overdispersed. We thus modelled this data using a zero-inflated negative binomial model (Ntzoufras 2009): 


$$
\begin{aligned}
& C_{i} \sim N B\left(\phi_{i}, r_{i}\right) \\
& \phi_{i}=\frac{r_{i}}{r_{i}+\lambda_{i}\left(1-\theta_{i}\right)} \\
& \theta_{i} \sim B\left(\psi_{i}\right) \\
& \ln \left(\lambda_{i}\right)=\alpha+\beta_{Z[i]}+\gamma_{F[i]}+\delta_{Z[i], F[i]}+\epsilon_{E[i], D[i]}+\zeta_{T[i]} \\
& \epsilon_{E[i], D[i]} \sim N\left(0, \sigma_{\epsilon}^{2}\right) \\
& \zeta_{T[i]} \sim N\left(0, \sigma_{\zeta}^{2}\right)
\end{aligned}
$$

260

where subscript $i$ indexes sampling events corresponding to a taxon (low-level phylogenetic

262 lumping, 12 groups) in a given enclosure on a given sampling day ( $\mathrm{n}=324$ sampling events), $\sim$ means "follows distribution", and $N B$ is the negative binomial distribution with success probability $\phi$ and 264 number of failures $r$. The $\theta$ latent variable for absence of organisms was modelled as a Bernoulli process (B distribution) with probability $\psi$.

266

Line 4 in Eq. (1) shows that we modelled positive (non-zero) counts $\lambda_{i}$ with a GLM including a natural logarithm (ln) link and a linear predictor in which $\alpha$ was an intercept, and $\beta$ described deviations from the intercept associated with zooplankton trophic group $Z$, which corresponded to

270 the six different grouping structures based on (i) body size (two $Z$ groups), (ii) antipredator behaviour (four $Z$ groups), (iii) body size and behaviour combined (five $Z$ groups), (iv) low-level

272 phylogenetic lumping (12 $Z$ groups), (v) medium-level phylogenetic lumping (10 $Z$ groups) and (vi) high-level phylogenetic lumping (four $Z$ groups).

$\gamma$ described deviations from the intercept associated with fish treatment $F$, which took either two 276 levels (fish absent $v s$. present) or 3 levels (fish absent $v s$. perch present $v s$. roach present). $\delta$ described deviations from the intercept due to the interaction between $Z$ and $F$. The model also 
278 included the normally-distributed random effect of enclosures $E$ on a given sampling date $D$ ( $\epsilon$ effect, $\quad n=9 \times 3=27$ levels), and of the low-level phylogenetic lumping $T$ ( $\zeta$ effect, $n=12$

280 levels), because these effects generated non-independence among observations but were not the primary focus of statistical inference (for further motivations to use random effects, see Kéry and 282 Schaub 2012). Note that $T$ was confounded with $Z$ for the low-level (12-group) phylogenetic lumping model, and $\zeta$ was thus omitted from this particular model.

We assessed the relative fit of the 12 different candidate models (six different $Z$ times two different

$286 \quad F \quad$ ) to the count data using statistical model selection. Specifically, we ranked models based on their parsimony, as measured by AIC computed as $\mathrm{AIC}=-2 \ln (L)+2 k$ and Bayesian information criterion 288 (BIC, also known as Schwarz's Bayesian criterion) computed as BIC $=-2 \ln (L)+\ln \left(n_{o b s}\right) k$, where $L$ is the likelihood of the estimated model, $k$ is the total number of parameters that are estimated in the

290 model and $n_{o b s}$ is the number of observations to which the model was fitted $(\mathrm{n}=324)$. The pseudo $\mathrm{R}^{2}$ of each model was computed from the linear regression of observations on fitted model values. The 292 statistical significance of fixed effect terms in the most parsimonious model (Table 3) was tested using likelihood ratio tests comparing the likelihood of the full model with that of a model omitting the focal 294 term.

296 Zooplankton body sizes

In addition to zooplankton counts, our dataset included body size measurements for crustaceans (see 298 above). We predicted fish predation to select for decreased body sizes in zooplankton, with the amplitude of body downsizing being proportional to the intensity of predation (Brooks and Dodson 
300 1965). To test this prediction, we modelled the effect of fish on natural log-transformed, individual zooplankton body sizes $S$ using a linear mixed model:

302

$$
\begin{aligned}
& S_{i} \sim N\left(\mu_{i}, \sigma^{2}\right) \\
& \mu_{i}=\alpha+\beta_{Z[i]}+\gamma_{F[i]}+\delta_{Z[i], F[i]}+\epsilon_{E[i], D[i]} \\
& \epsilon_{E[i], D[i]} \sim N\left(0, \sigma_{\epsilon}^{2}\right)
\end{aligned}
$$

304

where subscript $i$ indexes zooplankton individuals $(\mathrm{n}=3291$ individuals $), \quad N$ is the normal

306 (Gaussian) distribution, and $\mu$ and $\sigma^{2}$ are the mean and variance of $S_{i}$, respectively. Other parameters are as described in Eq. 1 and the grouping structure for $Z$ was that yielding the lowest

308 AIC in fitting model 1 to the count data. This structure was provided by the low-level taxonomic lumping (see Results) which explains why, compared to Eq. 1, the $\zeta_{T[i]}$ term is lacking in Eq. 2. We

310 modelled fish treatment $F$ using three levels (fish absent $v s$. perch present $v s$. roach present), which yielded a lower AIC (689 vs. 709) than using two levels (fish absent vs. present).

\section{Phytoplankton concentration}

314 We modelled chlorophyll $a$ concentration $P\left(\mu \mathrm{g} \mathrm{L}^{-1}\right)$, a proxy for phytoplankton concentration, using a linear mixed model:

$$
\begin{aligned}
& P_{i} \sim N\left(\mu_{i}, \sigma^{2}\right) \\
& \mu_{i}=\alpha+\beta_{F[i]}+\delta_{D[i]} \\
& \delta_{D[i]} \sim N\left(0, \sigma_{\delta}^{2}\right)
\end{aligned}
$$

where subscript $i$ indexes a lake enclosure $(n=9$ enclosures) on a given sampling date $(n=2$

318 sampling dates). Here also, a three-level fish treatment $F$ yielded a lower model AIC (61 vs. 74) than a two-levels $F$. Other subscripts are as described in Eq. 1. 
All statistics were performed in $\mathrm{R}$ version 3.6.0 ( $\mathrm{R}$ Core Team 2019). We fitted model 1 using the

322 glmmTMB ( ) function from the glmmTMB package (Brooks et al. 2017). Predictions with confidence intervals for model 1 were obtained using the predict.glmmTMB () function with a family =

324 nbinom2 argument. We fitted models 2 and 3 using the lmer () function from the lme 4 package (Bates et al. 2015) and obtained predictions with confidence intervals for these models using the

326 function sim () from the arm package (Gelman and Hill 2007). Significance tests of each term in the best-fitting version of model 1 (Table 3) were performed using the default anova ( ) function.

\section{Results}

\section{Zooplankton abundances and zooplankton trophic groups}

Overall, we found a relatively good agreement between our qualitative predictions and observed

332 response of zooplankton abundance to perch and roach (Table 1), indicating that prior literature knowledge is a reliable source of information to roughly predict trophic interactions. In particular,

334 perch and roach had remarkably different effects on zooplankton counts. For all of the six different zooplankton grouping structures, considering perch and roach as having different effects on

336 zooplankton counts strongly improved model fit (Table 2).

338 Our qualitative predictions of the effects of perch and roach on body-size based and behaviour-based zooplankton trophic groups were also relatively well supported by observations, except for the "Hide"

340 trophic group, which largely increased in response to roach when we predicted no response (Table 1, Behaviour-based - an increase which was mainly driven by Acanthocyclops, Fig. 1). This mismatch

342 between predictions and observations suggests that body size was a more parsimonious predictor of zooplankton numerical response to fish than behaviour. Accordingly, the body size-based zooplankton 
344 grouping structure ( $n=$ two zooplankton trophic groups) ranked better than the behaviour-based one ( $n$ $=$ three zooplankton trophic groups) in terms of both pseudo $\mathrm{R}^{2}$ and parsimony (Table 2).

Interestingly, the combined body size- and behaviour-based zooplankton grouping structure, in which 348 "no escape" zooplankters were categorized as either large- or small-bodied ( $n=$ four zooplankton trophic groups), was more parsimonious in terms of both AIC and BIC than the size-dependent model

350 (Table 2), indicating that body size becomes a more important predictor of trophic interactions when behaviour can not deter predators. However, here also our predictions were poorly validated for "hide" 352 zooplankton strategists, which abundances were mainly unchanged by perch when we predicted a strong increase, and were strongly increased by roach when we predicted a moderate increase (Table 1,

354 Body size- and Behaviour-based).

356 Body size- or/and behaviour-based zooplankton grouping structures were largely outperformed by phylogeny-based structures in terms of both pseudo $\mathrm{R}^{2}$ and parsimony (Table 2). This was true for both

358 the low (12 zooplankton trophic groups) and medium taxonomic-lumping models (10 zooplankton trophic groups), but not for the high taxonomic-lumping model (four zooplankton trophic groups),

360 which was outperformed by the combined size- and behaviour-based zooplankton grouping structure (Table 2). Note that the lower parsimony of the low taxonomic lumping model (Table 2) was not due to 362 the loss of a random-effect term ( $\zeta$ in Eq. 1), which penalized AIC and BIC through increasing the number of model parameters (a random intercept counts as one parameter when the fixed effect 364 counted as 12 parameters).

366 A significant fish treatment-by-zooplankton group interaction (Table 3) indicated that the high performance of phylogeny in predicting zooplankton response to fish was explained by taxon-specific 
368 patterns that could not accurately fit into body size- and behaviour-based trophic groups. Accordingly, among large-bodied zooplankters some decreased in response to fish (e.g., Ceriodaphnia, Daphnia),

370 while others increased (e.g., Acanthocyclops) or were unaffected (e.g., Diaphanosoma, Fig. 1). Similarly, among "hide" strategists the strongly positive effect of fish on Ancanthocyclops did not 372 match with the absence of any effect on Thermocyclops (Fig. 1), indicating that similarly-behaving prey genera did not respond similarly to their joint predators.

Finally, we found that accounting for copepod ontogenetic stages was crucial to the performance of

376 phylogeny at a high resolution, but not at a medium or low resolution (Table S1), indicating ontogenyby-phylogeny interactions in copepod response to fish. Accordingly, Calanoids (i.e. Eudiaptomus) were

378 similarly affected by fish at the nauplius and copepodite stages, while among Cyclopoids of either stages only copepodites of Acanthocyclops responded to fish (Fig. 1).

\section{Crustacean zooplankton body size}

382 There was a highly significant interaction between phylogeny and fish treatments on crustacean zooplankton body sizes (Fig. 2, LR-test, Chisq $=189.9, \mathrm{df}=12, \mathrm{p}<0.001$ ), which tended to mirror the

384 phylogeny-fish interaction on zooplankton abundances described above. Specifically, pairwise $t$-tests (not shown) revealed that perch significantly decreased body sizes in all the seven crustacean genera

386 but Daphnia (which were too few in fish treatments to draw statistical inference), while roach significantly reduced body sizes in Bosmina, Ceriodaphnia, Diaphanosoma and Acanthocyclops only

388 (Fig. 2).

\section{Phytoplankton}


Fish induced a trophic cascade in which chlorophyll $a$ concentration almost doubled in presence of

392 roach and almost tripled in presence of perch (Table 1, Fig. 3). This result fits with the a priori predictions that both body size and hunting mode were making perch more efficient zooplankton 394 predators than roach under the conditions of our experiment (Table 1).

\section{Discussion}

398 To our knowledge, the relative contributions of body size, antipredator behaviour, and phylogeny in determining trophic interactions has not been previously assessed in a particular system. By using

400 freshwater zooplankton communities as model, we showed that body size alone is a better predictor of trophic interactions than behaviour alone, but that prey body size and behaviour combined

402 outperformed both. We further found that zooplankton phylogeny was a far better predictor of zooplankton trophic interactions than body size and behaviour combined when phylogeny was

404 informed at a high (genus-level) to medium (family-level) resolution, but not at a low resolution (highlevel taxonomic groups). These results have several general implications for food-web research.

Recent studies have highlighted that the interplay between predator hunting mode and prey antipredator

408 behaviour is an important driver of trophic interactions (Schmitz 2008, Belgrad and Griffen 2016). Our results suggest, however, that zooplankton behaviour is less important than zooplankton body size in

410 driving trophic interactions in food webs of shallow lakes. Our results are in line with the well-known importance of body size in driving trophic interactions in aquatic systems (Elton 1927, Cohen et al.

412 2003, Emmerson and Raffaelli 2004, Woodward et al. 2005, Brose et al. 2006). Further studies would be needed to test whether our results hold true also in terrestrial systems, where body size is expected 
414 to be less important than in aquatic communities to determining trophic interactions (Shurin et al. 2006).

Our results testify for the importance of phenotypic traits in driving the strength of trophic interactions

418 and ecosystem function at the plankton level. At the fish level also, traits were involved in driving the differential effects of perch and roach. Despite identical biomasses, perch more severely depleted 420 zooplankters than roach, and induced a trophic cascade that was more than twice as large as the trophic cascade induced by roach (Table 1 effect sizes, Fig. 3). Our experimental design, however, varied fish

422 identity in parallel with fish density and body size (owing to a constant fish biomass), but also in parallel with fish hunting mode. Hence, we could not rank the contributions of body size (and related

424 fish density) and hunting modes in controlling the differential predatory effects of perch and roach on zooplankters. The strength of ecological interactions is classically considered as driven by the biomass

426 of interacting species (Oksanen et al. 1981, Carpenter et al. 1985, Brett and Goldman 1996, Jeppesen et al. 2003), a rule dubbed "law of mass action". Our result demonstrate that this paradigm is not 428 sufficient to predict the strength of ecological interactions, in line with previous studies that demonstrated the importance of fish body size in determining the strength of their induced trophic 430 cascades (Persson et al. 2003, Shackell et al. 2010, Renneville et al. 2016).

432 Despite the important role of body size and behaviour in determining zooplankton trophic groups, our results bring support to the previous contention that phylogeny is an even more powerful predictor of

434 food-web structure (Cattin et al. 2004, Naisbit et al. 2012). Phylogenetic relatedness is a surrogate for a host of unknown or poorly documented traits that potentially influence trophic interactions, but that are

436 not captured by body size or behavioural strategies such as, for instance, food preferences, diel activity cycles, body shape, mechanical defences (e.g. spines, helmets, carapaces), colouration or camouflages 
438 (Boukal 2014). However, in our study phylogeny at a high resolution was the best predictor of the trophic structure only when combined with copepod ontogeny (i.e., nauplius vs. copepodite stages,

440 Table S1), which is another synthetic proxy encapsulating information on body-size, behaviour or feeding preferences (Werner and Gilliam 1984). Ontogeny further accounts for the fact that juveniles

442 are often more abundant than adults. Hence, phylogeny does not capture all the traits that determine trophic niches, which are probably best predicted by combining phylogeny with other, non-redundant

444 and synthetic sources of information. We further found that the power of phylogeny in predicting trophic structure degraded at a low resolution, indicating that body size combined with behaviour (but

446 not alone) should be preferred predictors of trophic niches when information on phylogenetic resolution is limited. However, information on hunting mode or antipredator behaviour may also be

448 limited, or dependent on detailed phylogenetic information (e.g., species among the same genus may vary in behaviour), in which case the most readily implementable predictor of trophic niches remains 450 body size.

452 Our study illustrates how food-web perturbation experiments may be used to infer trophic interactions. Our approach included two steps. First, we formed qualitative predictions from literature knowledge 454 for the effects of fish manipulation on the abundance of planktonic organisms while assuming trophic interactions to be dependent on body size only, on antipredator behaviour only, or on body size and

456 behaviour combined (Table 1). Second, we statistically modelled the effects of fish manipulation on plankton grouped into body size- and behavioural trophic groups (alone or mixed) and into 458 phylogenetic trophic groups (combined with ontogeny). This modelling step allowed us to (i) compute effect sizes to which our qualitative predictions could be compared (Table 1), and (ii) to quantify the 460 relative efficiencies of body size, behaviour and phylogeny in predicting plankton response to fish manipulation (Table 2). 
Compared to classical approaches to study food-web structure, this experimental approach has the 464 strong advantage not to require any a priori data on observed trophic networks. High-quality of such food-web data are costly to acquire and, often, only report presence or absence of pairwise trophic links 466 with no information on the strength or potential transience of the links. In contrast, our approach allowed us to quantify the realized effects of controlled changes in the trophic structure. A draback of 468 our approach, however, is that realized affects do not separate direct from indirect effects of trophic interactions. Additionally, our approach still required prior knowledge to group organisms into separate

470 body size and behavioural trophic groups. Body size data are readily available, but pertinent cutoffs for body size binning values are not necessarily easy to define. Here, we defined these cutoff values for

472 zooplankton body sizes arbitrarily, based on the observed body-size distributions. Predator hunting mode and prey antipredator behaviour might also not be readily available from the literature, or simply

474 for the taxonomic level at which organisms were determined. Therefore, just as approaches relying on a priori food-web data, our approach might be limited to organisms for which substantial prior 476 knowledge is available.

478 To conclude, our results illustrate how experiments aiming at exploring community response to topdown perturbations may also be used to infer the rules that control food-web assembly. In taking this 480 approach, we found that body size, behaviour, phylogeny and ontogeny were all pertinent predictors of trophic niches in zooplankton food webs of Lake Créteil, but that phylogeny combined with ontogeny 482 was most powerful. This result highlights that, beyond the important roles of body size and behaviour, other traits are involved in structuring food webs. Uncovering these traits remains highly needed if we 484 are to understand the ecological mechanisms that shape trophic networks and support biodiversity. 
AFNOR 1990. Eaux: méthodes d'essai. - Association française de normalisation.

Angeli, N. et al. 1995. Diel patterns of feeding and vertical migration in daphnids and diaptomids during the clear water phase in Lake Geneva (France). - Hydrobiologia 300: 163-184.

Bates, D. et al. 2015. Fitting linear mixed-effects models using lme4. - J. Stat. Softw. 67: 1-48.

Belgrad, B. A. and Griffen, B. D. 2016. Predator-prey interactions mediated by prey personality and predator hunting mode. - Proc. R. Soc. B Biol. Sci. 283: 20160408.

Bertolo, A. et al. 1999a. Effects of physical refuges on fish-plankton interactions. - Freshw. Biol. 41: 795-808.

Bertolo, A. et al. 1999b. Scaling food chains in aquatic mesocosms: do the effects of depth override the effects of planktivory? - Oecologia 121: 55-65.

Bertolo, A. et al. 2000. Plankton dynamics in planktivore- and piscivore-dominated mesocosms. - Arch. Für Hydrobiol. 147: 327-349.

Bolker, B. et al. 2003. Connecting theoretical and empirical studies of trait-mediated interactions. Ecology 84: 1101-1114.

Boukal, D. S. 2014. Trait- and size-based descriptions of trophic links in freshwater food webs: current status and perspectives. - J. Limnol. s1: 171-185.

Brett, M. T. and Goldman, C. R. 1996. A meta-analysis of the freshwater trophic cascade. - Proc. Natl. Acad. Sci. U. S. A. 93: 7723-7726.

Brooks, J. L. and Dodson, S. I. 1965. Predation, body size, and composition of plankton. - Science 150: 28.

Brooks, M. E. et al. 2017. glmmTMB balances speed and flexibility among packages for zero-inflated generalized linear mixed modeling. - R J. 9: 378-400.

Brose, U. et al. 2006. Consumer-resource body-size relationships in natural food webs. - Ecology 87: 2411-2417.

Carpenter, S. R. et al. 1985. Cascading trophic interactions and lake productivity. - BioScience 35: 634-639.

Cattin, M.-F. et al. 2004. Phylogenetic constraints and adaptation explain food-web structure. - Nature 427: 835-839.

Chang, K.-H. and Hanazato, T. 2003. Vulnerability of cladoceran species to predation by the copepod Mesocyclops leuckarti: laboratory observations on the behavioural interactions between predator and prey. - Freshw. Biol. 48: 476-484. 
Cohen, J. E. et al. 1993. Body sizes of animal predators and animal prey in food webs. - J. Anim. Ecol. 62: $67-78$.

Cohen, J. E. et al. 2003. Ecological community description using the food web, species abundance, and body size. - Proc. Natl. Acad. Sci. 100: 1781-1786.

Diehl, S. 1988. Foraging efficiency of three freshwater fishes: effects of structural complexity and light. - Oikos 53: 207-214.

Doulka, E. and Kehayias, G. 2011. Seasonal vertical distribution and diel migration of zooplankton in a temperate stratified lake. - Biologia (Bratisl.) 66: 308-319.

Elton, C. S. 1927. Animal ecology. - Macmillan Co.

Emmerson, M. C. and Raffaelli, D. 2004. Predator-prey body size, interaction strength and the stability of a real food web. - J. Anim. Ecol. 73: 399-409.

Gauzens, B. et al. 2013. Food-web aggregation, methodological and functional issues. - Oikos 122: 1606-1615.

Gelman, A. and Hill, J. 2007. Data analysis using regression and multilevel-hierarchical models. Cambridge University Press.

Helenius, L. K. et al. 2015. Strategies of zooplanktivory shape the dynamics and diversity of littoral plankton communities: a mesocosm approach. - Ecol. Evol. 5: 2021-2035.

Hulot, F. D. et al. 2014. Differential responses of size-based functional groups to bottom-up and topdown perturbations in pelagic food webs: a meta-analysis. - Oikos 123: 1291-1300.

Jeppesen, E. et al. 2003. The impact of nutrient state and lake depth on top-down control in the pelagic zone of lakes: a study of 466 lakes from the temperate zone to the Arctic. - Ecosystems 6: 313325 .

Kerfoot, W. C. 1978. Combat between predatory copepods and their prey: Cyclops, Epischura, and Bosmina. - Limnol. Oceanogr. 23: 1089-1102.

Kéry, M. and Schaub, M. 2012. Bayesian population analysis using WinBUGS. - Academic Press.

Kestemont, P. et al. 2015. Biology and culture of percid fishes. - Springer.

Lacroix, G. and Lescher-Moutoué, F. 1995. Spatial patterns of planktonic microcrustaceans in a small shallow lake. - Hydrobiologia 300: 205-217.

Lacroix, G. et al. 1996. Trophic interactions, nutrient supply, and the structure of freshwater pelagic food webs. - In: Hochberg, M. E. and Barbault, R. (eds), Aspects of the genesis and maintenance of biological diversity. 1st ed.n. Oxford University Press, pp. 162-179.

Lazzaro, X. et al. 2009. Predator foraging behaviour drives food-web topological structure. - J. Anim. Ecol. 78: 1307-1317. 
Lieschke, J. A. and Closs, G. P. 1999. Regulation of zooplankton composition and distribution by a zooplanktivorous fish in a shallow, eutrophic floodplain lake in south east Australia. - Arch. Für Hydrobiol. 146: 397-412.

Naisbit, R. E. et al. 2012. Phylogeny versus body size as determinants of food web structure. - Proc. R. Soc. B Biol. Sci. 279: 3291.

Ntzoufras, I. 2009. Bayesian modeling using WinBUGS. - Wiley.

Nurminen, L. et al. 2010. Feeding efficiency of planktivorous perch Perca fluviatilis and roach Rutilus rutilus in varying turbidity: an individual-based approach. - J. Fish Biol. 76: 1848-1855.

Oksanen, L. et al. 1981. Exploitation ecosystems in gradients of primary productivity. - Am. Nat. 118: $240-261$.

Papińska, K. 1981. Occurrence of filtering crustacea in the near-bottom and pelagial waters of the Mikolajskie Lake. - Hydrobiologia 83: 411-418.

Papińska, K. and Prejs, K. 1979. Crustaceans of the near-bottom water and bottom sediments in 24 Masurian Lakes with special consideration to cyclopoid copepods. - Ekol. Pol. 28: 219-230.

Persson, L. et al. 2003. Gigantic cannibals driving a whole-lake trophic cascade. - Proc. Natl. Acad. Sci. 100: 4035-4039.

Peterka, J. and Matěna, J. 2009. Differences in feeding selectivity and efficiency between young-ofthe-year European perch (Perca fluviatilis) and roach (Rutilus rutilus) - field observations and laboratory experiments on the importance of prey movement apparency vs. evasiveness. Biologia (Bratisl.) 64: 786-794.

Peterka, J. and Matěna, J. 2011. Feeding behaviour determining differential capture success of evasive prey in underyearling European perch (Perca fluviatilis L.) and roach (Rutilus rutilus (L.)). Hydrobiologia 661: 113-121.

Polis, G. A. and Winemiller, K. O. 1996. Food webs: integration of patterns \& dynamics. - Springer US.

R Core Team 2019. R: a language and environment for statistical computing. - R Foundation for Statistical Computing.

Rall, B. C. et al. 2012. Universal temperature and body-mass scaling of feeding rates. - Philos. Trans. R. Soc. Lond. B Biol. Sci. 367: 2923-2934.

Reid, J. W. and Williamson, C. E. 2010. Copepoda. - In: Thorp, J. H. and Covich, A. P. (eds), Ecology and classification of north American freshwater invertebrates. Academic Press, pp. 829-899.

Renneville, C. et al. 2016. Morphological drivers of trophic cascades. - Oikos 125: 1193-1202. 
Rohr, R. P. et al. 2017. Statistical approaches for inferring and predicting food-web architecture. - In: Moore, J. C. et al. (eds), Adaptive food webs: stability and transitions of real and model ecosystems. Cambridge University Press, pp. 178-192.

Sakamoto, M. and Hanazato, T. 2008. Antennule shape and body size of Bosmina: key factors determining its vulnerability to predacious Copepoda. - Limnology 9: 27-34.

Schmitz, O. J. 2008. Effects of predator hunting mode on grassland ecosystem function. - Science 319: 952.

Schmitz, O. J. et al. 2004. Trophic cascades: the primacy of trait-mediated indirect interactions. - Ecol. Lett. 7: 153-163.

Shackell, N. L. et al. 2010. Decline in top predator body size and changing climate alter trophic structure in an oceanic ecosystem. - Proc. R. Soc. Lond. B Biol. Sci. 277: 1353-1360.

Shurin, J. B. et al. 2006. All wet or dried up? Real differences between aquatic and terrestrial food webs. - Proc. R. Soc. Lond. B Biol. Sci. 273: 1-9.

Titelman, J. and Fiksen, Ø. 2004. Ontogenetic vertical distribution patterns in small copepods: field observations and model predictions. - Mar. Ecol. Prog. Ser. 284: 49-63.

Van Den Berg, C. et al. 1993. Zooplankton feeding in common bream (Abramis Brama), white bream (Blicca Bjoerkna) and roach (Rutilus Rutilus): experiments, models and energy Intake. - Neth. J. Zool. 44: 15-42.

Werner, E. E. and Gilliam, J. F. 1984. The ontogenetic niche and species interactions in size-structured populations. - Annu. Rev. Ecol. Syst. 15: 393-425.

Werner, E. E. and Peacor, S. D. 2003. A review of trait-mediated indirect interactions in ecological communities. - Ecology 84: 1083-1100.

Williams, A. E. and Moss, B. 2003. Effects of different fish species and biomass on plankton interactions in a shallow lake. - Hydrobiologia 491: 331-346.

Williamson, C. E. 1983. Behavioral interactions between a cyclopoid copepod predator and its prey. - J. Plankton Res. 5: 701-711.

Woodward, G. et al. 2005. Body size in ecological networks. - Trends Ecol. Evol. 20: 402-409. 
488 Table 1. Qualitative predictions and observed effect sizes for the numerical response to perch and roach of zooplankton taxa assigned to trophic groups, based either on body size only, on 490 antipredator behaviour only, or on body size and behaviour combined. Qualitative predictions were formed based on prior literature knowledge. Predicted - $(--),+(++)$ or $=$ represent a negative 492 (very negative), positive (very positive) or non-existent numerical response by zooplankton to fish, respectively. Observed $\%$ effects sizes were computed as $100\left(\mu_{F}-\mu_{C}\right) / \mu_{C}$, where $\mu_{F}$ and $\mu_{C}$ are

494 mean plankton counts in fish and control treatments, respectively, as estimated from statistical models described in Eq. 1 (zooplankton) and Eq. 3 (phytoplankton).

\begin{tabular}{|c|c|c|c|c|c|c|c|}
\hline $\begin{array}{l}\text { Basis for } \\
\text { trophic } \\
\text { groups }\end{array}$ & $\begin{array}{l}\text { Zooplancton } \\
\text { groups }\end{array}$ & $\begin{array}{l}\text { Zooplankton } \\
\text { Phyla }\end{array}$ & $\begin{array}{l}\text { Predicted } \\
\text { Perch } \\
\text { ef ect }\end{array}$ & $\begin{array}{l}\text { Observed } \\
\text { Perch } \\
\text { ef ect }(\%)\end{array}$ & $\begin{array}{l}\text { Predicted } \\
\text { Roach } \\
\text { ef ect }\end{array}$ & $\begin{array}{l}\text { Observed } \\
\text { Roach } \\
\text { ef ect }(\%)\end{array}$ & Explanation of predicted f $\dot{s} h$ ef ects \\
\hline \multirow{3}{*}{ Body size } & Large zooplankton & $\begin{array}{l}\text { Asplanchna, Ceriodaphnia, } \\
\text { Daphnia, Diaphanosoma, } \\
\text { Bosmina, Acanthocyclops, } \\
\text { Thermocyclops, } \\
\text { Eudiaptomus }\end{array}$ & -- & -81.3 & - & -22.6 & $\begin{array}{c}\text { Higher cumulated attack rate in perch than } \\
\text { roach. }\end{array}$ \\
\hline & Small zooplankton & $\begin{array}{l}\text { Hexarthra, Polyarthra, nauplii } \\
\text { of Calanoids, nauplii of } \\
\text { Cyclopoids }\end{array}$ & ++ & 39.5 & + & 23.1 & $\begin{array}{c}\text { Depletion of large-bodied zooplankton } \\
\text { favours competitively dom inated smaller } \\
\text { zooplankton. }\end{array}$ \\
\hline & Phytoplankton & & ++ & 190.0 & + & 82.2 & $\begin{array}{l}\text { Trophic cascade driven by decreased grazing } \\
\text { from large-bodied zooplankton. }\end{array}$ \\
\hline \multirow{4}{*}{ Behaviour } & Flee & $\begin{array}{c}\text { Bosmina, Diaphanosoma, } \\
\text { Eudiaptomus }\end{array}$ & - & -91.2 & $=$ & 6.7 & $\begin{array}{l}\text { Fleeing strategy by zooplankton inef Eient } \\
\text { against perch but ef Eient against roach. }\end{array}$ \\
\hline & Hide & $\begin{array}{l}\text { Acanthocyclops, } \\
\text { Therm ocyclops, nauplii of } \\
\text { Cyclopoids }\end{array}$ & $=$ & 8.3 & $=$ & 72.2 & $\begin{array}{c}\text { "Hide" strategy by zooplankton ef tient } \\
\text { against both perch and roach. }\end{array}$ \\
\hline & No escape & $\begin{array}{c}\text { Asplanchna, Ceriodaphnia, } \\
\text { Daphnia, Hexarthra, } \\
\text { Polyarthra, nauplii of } \\
\text { Calanoids }\end{array}$ & - & -58.8 & -- & -42.1 & $\begin{array}{l}\text { "No escape" zooplankton strategists are } \\
\text { similarly depleted by perch and roach. }\end{array}$ \\
\hline & Phy toplankton & & ++ & 190.0 & + & 82.2 & $\begin{array}{c}\text { Stronger trophic cascade when both "no } \\
\text { escape" and "f eeing" strategists are } \\
\text { depleted. }\end{array}$ \\
\hline \multirow{5}{*}{$\begin{array}{l}\text { Body size } \\
\quad \text { and } \\
\text { Behaviour }\end{array}$} & Flee & $\begin{array}{c}\text { Bosmina, Diaphanosoma, } \\
\text { Eudiaptomus }\end{array}$ & -- & -90.9 & $=$ & 9.2 & $\begin{array}{l}\text { Response of "f keing" zooplankton } \\
\text { strategists is driven by behaviour only. }\end{array}$ \\
\hline & Hide & $\begin{array}{c}\text { Acanthocyclops, } \\
\text { Therm ocyclops, nauplii of } \\
\text { Cyclopoids }\end{array}$ & ++ & 8.3 & + & 70.8 & $\begin{array}{l}\text { "Hide" zooplankton strategists are } \\
\text { carnivorous and benef t from increasing } \\
\text { abundances of small, no escape } \\
\text { zooplankters on which they feed. }\end{array}$ \\
\hline & Large, no escape & $\begin{array}{l}\text { Asplanchna, Daphnia, } \\
\text { Ceriodaphnia }\end{array}$ & - & -92.1 & - & -69.0 & $\begin{array}{l}\text { Response of "no escape" plankton } \\
\text { strategists is driven by body size only. }\end{array}$ \\
\hline & Small, no escape & $\begin{array}{l}\text { Hexarthra, Polyarthra, nauplii } \\
\text { of Calanoids }\end{array}$ & ++ & 70.4 & + & 39.1 & $\begin{array}{l}\text { Response of "no escape" plankton } \\
\text { strategists is driven by body size only. }\end{array}$ \\
\hline & Phytoplankton & & ++ & 190.0 & + & 82.2 & $\begin{array}{c}\text { Trophic cascade driven by decreased grazing } \\
\text { from large-bodied zooplankton and stronger } \\
\text { when both "no escape" and "f eeing" } \\
\text { zooplankters are depleted. }\end{array}$ \\
\hline
\end{tabular}


498 Table 2. Fit of the 12 candidate models to zooplankton count data. Columns "Candidate grouping structure" and "Separate perch-roach effects" correspond to zooplankton trophic groups $Z$ and fish 500 groups $F$, respectively, as described in Eq. 1. Non-shaded lines correspond to models that included a separate effect for perch and roach (i.e., three-level $F$ ). Shaded lines correspond to models that did 502 not include a separate effect for perch and roach (i.e., two-level $F$ ). Df gives the number of parameters in the model. Delta AIC compares each model to the lowest-AIC model.

504

\begin{tabular}{|c|c|c|c|c|c|c|c|}
\hline $\begin{array}{l}\text { Candidate zooplankton } \\
\text { grouping structure }\end{array}$ & $\begin{array}{l}\text { Separate Perch- } \\
\text { Roach effects }\end{array}$ & df & Deviance & Pseudo $\mathbf{R}^{2}$ & BIC & AIC & Delta AIC \\
\hline \multirow{2}{*}{$\begin{array}{l}\text { Phylogeny and ontogeny } \\
\text { High resolution }\end{array}$} & Yes & 39 & 2456.0 & 0.67 & 2681 & 2534 & 0 \\
\hline & No & 27 & 2567.6 & 0.50 & 2724 & 2622 & 88 \\
\hline \multirow{2}{*}{$\begin{array}{l}\text { Phylogeny and ontogeny } \\
\text { Medium resolution }\end{array}$} & Yes & 31 & 2507.1 & 0.66 & 2704 & 2575 & 41 \\
\hline & No & 24 & 2612.2 & 0.50 & 2751 & 2660 & 126 \\
\hline \multirow{2}{*}{ Body size x Behaviour } & Yes & 16 & 2597.2 & 0.59 & 2690 & 2629 & 95 \\
\hline & No & 12 & 2660.9 & 0.50 & 2730 & 2685 & 151 \\
\hline \multirow{2}{*}{$\begin{array}{l}\text { Phylogeny and ontogeny } \\
\text { Low resolution }\end{array}$} & Yes & 16 & 2638.1 & 0.57 & 2731 & 2670 & 136 \\
\hline & No & 12 & 2675.3 & 0.52 & 2745 & 2699 & 165 \\
\hline \multirow{2}{*}{ Body size } & Yes & 10 & 2650.6 & 0.59 & 2708 & 2671 & 137 \\
\hline & No & 8 & 2691.2 & 0.50 & 2737 & 2707 & 173 \\
\hline \multirow{2}{*}{ Behaviour } & Yes & 13 & 2660.6 & 0.50 & 2736 & 2687 & 153 \\
\hline & No & 10 & 2704.7 & 0.46 & 2762 & 2725 & 191 \\
\hline
\end{tabular}

506 
Table 3. Significance tests for focal terms in models 1 (zooplankton counts) and 2 (crustacean

510 zooplankton body sizes). In fitting models 1 and 2 , we used the fish $F$ and zooplankton $Z$ grouping structures that provided the best fit (i.e., $F=3$ groups from fish absent vs. perch present

512 vs. roach present, and $Z=12$ groups from a low-level lumping, high resolution phylogeny combined with copepod ontogeny, see Table 2).

514

\begin{tabular}{cccccc}
\hline Effect & Parameter & Model & Chisq & Df & P-value \\
\hline Zooplankton group & \multirow{2}{*}{$*$} & 1 & 676.0 & 33 & $<\mathbf{0 . 0 0 0 1}$ \\
& & 2 & 2772.1 & 18 & $<0.0001$ \\
Fish treatment & $\mathrm{V}$ & 1 & 205.9 & 24 & $<\mathbf{0 . 0 0 0 1}$ \\
Fish treatment $\mathrm{x}$ & & 2 & 230.4 & 14 & $<0.0001$ \\
Zooplankton group & $\delta$ & 1 & 190.4 & 22 & $<\mathbf{0 . 0 0 0 1}$ \\
\hline
\end{tabular}

516 


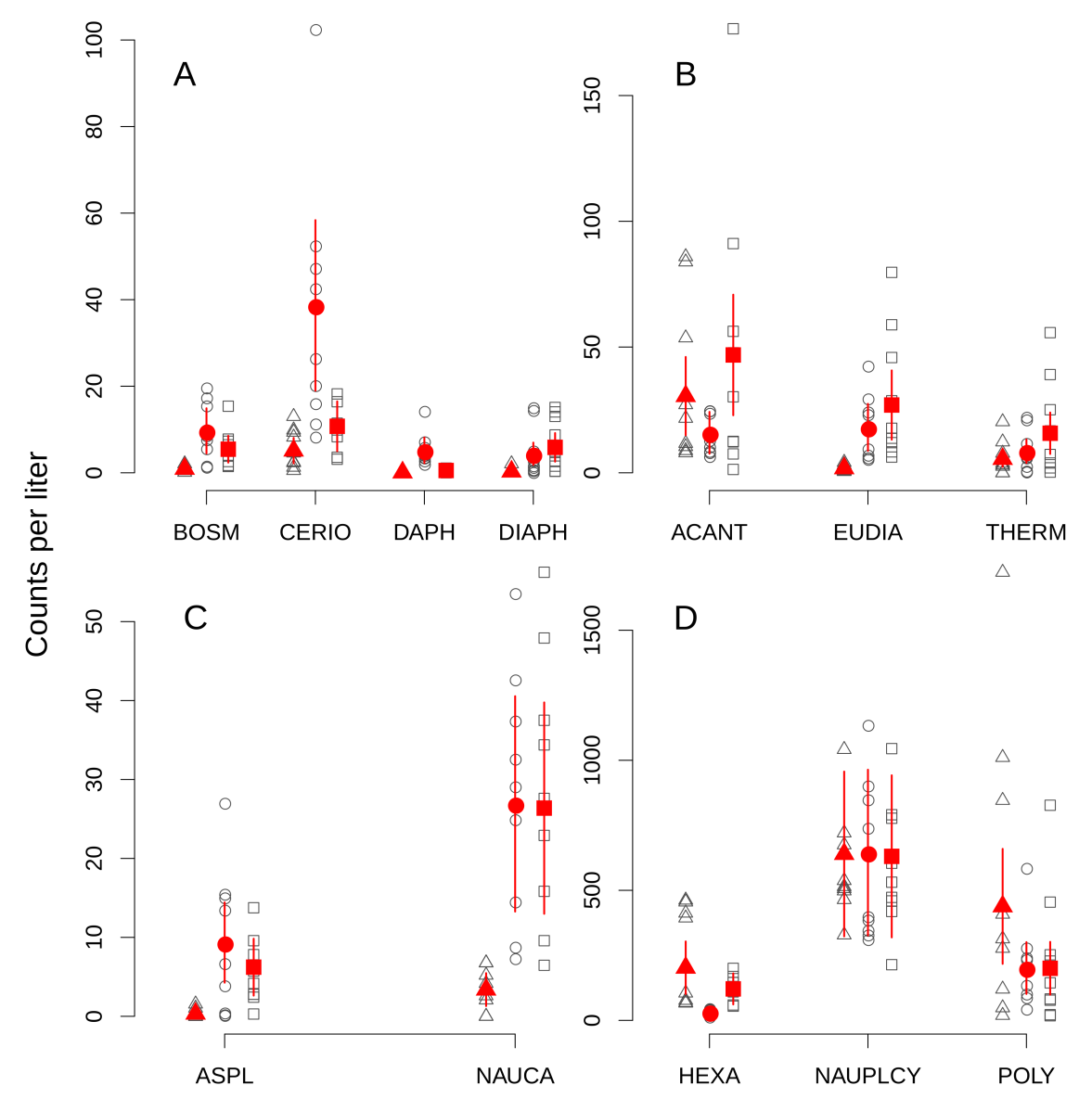

Fig. 1. Zooplankton numerical response to fish treatments. Filled, red symbols show mean 520 predicted counts with $95 \%$ confidence intervals as predicted from model 1. For a sampling datespecific equivalent of this figure, see Fig. S1. Circles: fishless control treatment; triangles: perch 522 treatment, squares: roach treatment. Open, gray symbols show the raw data. Zooplankton taxa were separated among A: Cladocerans $(\mathrm{BOSM}=$ Bosmina longirostris, CERIO = Ceriodaphnia pulchella 524 and C. quadrangula, DAPH = Daphnia longispina complex, DIAPH = Diaphanosoma brachyurum), B: Copepodite stages (ACANT = Acanthocyclops robustus, EUDIA: Eudiaptomus gracilis, THERM = 526 Thermocyclops crassus and T. oithonoides), C: NAUCA = nauplii of Calanoids, ASPL = Asplanchna girodi and $A$. priodonta, D: NAUPLCY = nauplii of Cyclopoids, HEXA = Hexarthra mira, POLY = 528 Polyarthra sp. 


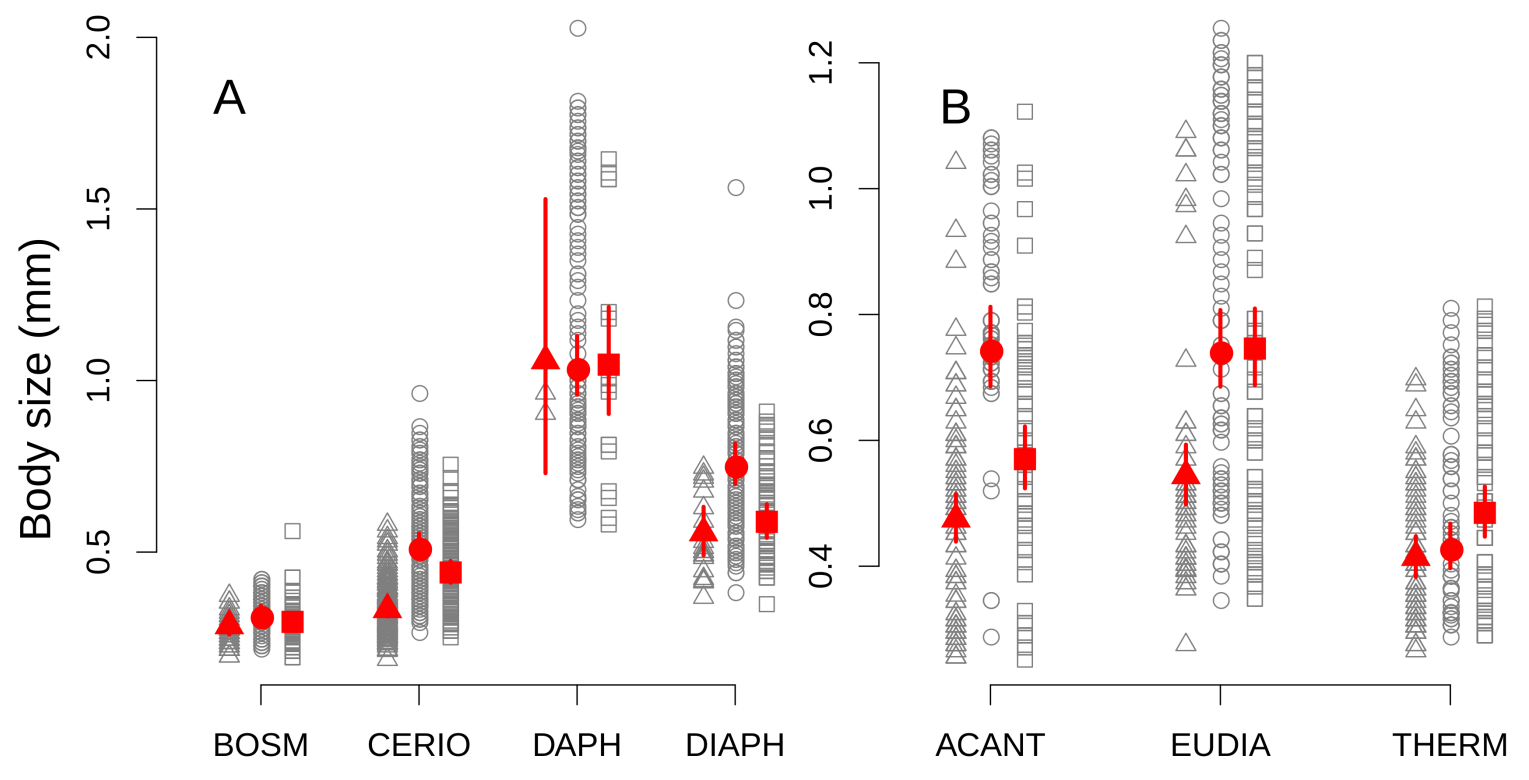

532 Fig. 2. Body-size response of crustacean zooplankton to fish treatments. Filled, red symbols show mean predicted counts with $95 \%$ confidence intervals as predicted from model 2. Circles: fishless

534 control treatment; triangles: perch treatment, squares: roach treatment. Open, gray symbols show the raw data. Zooplankton taxa were separated among A: Cladocerans (BOSM = Bosmina longirostris,

$536 \mathrm{CERIO}=$ Ceriodaphnia pulchella and C. quadrangula, DAPH = Daphnia longispina complex, DIAPH = Diaphanosoma brachyurum), B: Copepodite stages of copepods (ACANT = Acanthocyclops 538 robustus, EUDIA: Eudiaptomus gracilis, THERM = Thermocyclops crassus and T. oithonoides). 


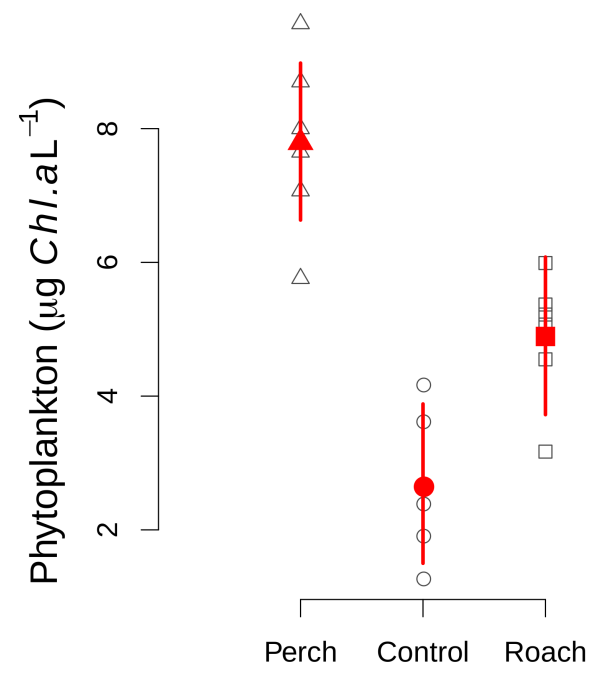

544 Fig. 3. Phytoplankton response to fish treatments. Filled, red symbols show mean predicted counts with 95\% confidence intervals as predicted from model 2. Circles: fishless control treatment; triangles:

546 perch treatment, squares: roach treatment. Open, gray symbols show the raw data. 
550 Table S1. Fit of ontogeny-free phylogeny-based models to zooplankton count data. Other models appear shaded, are as in Table 2 and are provided to ease comparison. Models were ranked 552 according to their AIC score. All models included a 3-level fish treatment considering fish absent $v s$. perch present $v s$. roach present.

\begin{tabular}{|c|c|c|c|c|c|c|}
\hline $\begin{array}{c}\text { Candidate food-web } \\
\text { Model }\end{array}$ & df & Deviance & Pseudo $\mathbf{R}^{2}$ & $\mathrm{BIC}$ & AlC & Delta AIC \\
\hline $\begin{array}{l}\text { Phylogeny and ontogeny } \\
\text { High resolution }\end{array}$ & 39 & 2456 & 0.67 & 2681 & 2534 & 0 \\
\hline $\begin{array}{l}\text { Phylogeny and ontogeny } \\
\text { Medium resolution }\end{array}$ & 34 & 2507 & 0.66 & 2704 & 2575 & 41 \\
\hline $\begin{array}{l}\text { Phylogeny alone } \\
\text { Medium resolution }\end{array}$ & 28 & 2528 & 0.62 & 2690 & 2584 & 50 \\
\hline Body size $x$ Behaviour & 16 & 2597 & 0.59 & 2690 & 2629 & 95 \\
\hline $\begin{array}{l}\text { Phylogeny alone } \\
\text { High resolution }\end{array}$ & 33 & 2598 & 0.41 & 2789 & 2664 & 130 \\
\hline $\begin{array}{l}\text { Phylogeny alone } \\
\text { Low resolution }\end{array}$ & 13 & 2644 & 0.54 & 2719 & 2670 & 136 \\
\hline $\begin{array}{l}\text { Phylogeny and ontogeny } \\
\text { Low resolution }\end{array}$ & 16 & 2638 & 0.57 & 2731 & 2670 & 136 \\
\hline Body size & 10 & 2651 & 0.59 & 2708 & 2671 & 137 \\
\hline Behaviour & 13 & 2661 & 0.50 & 2736 & 2687 & 153 \\
\hline
\end{tabular}



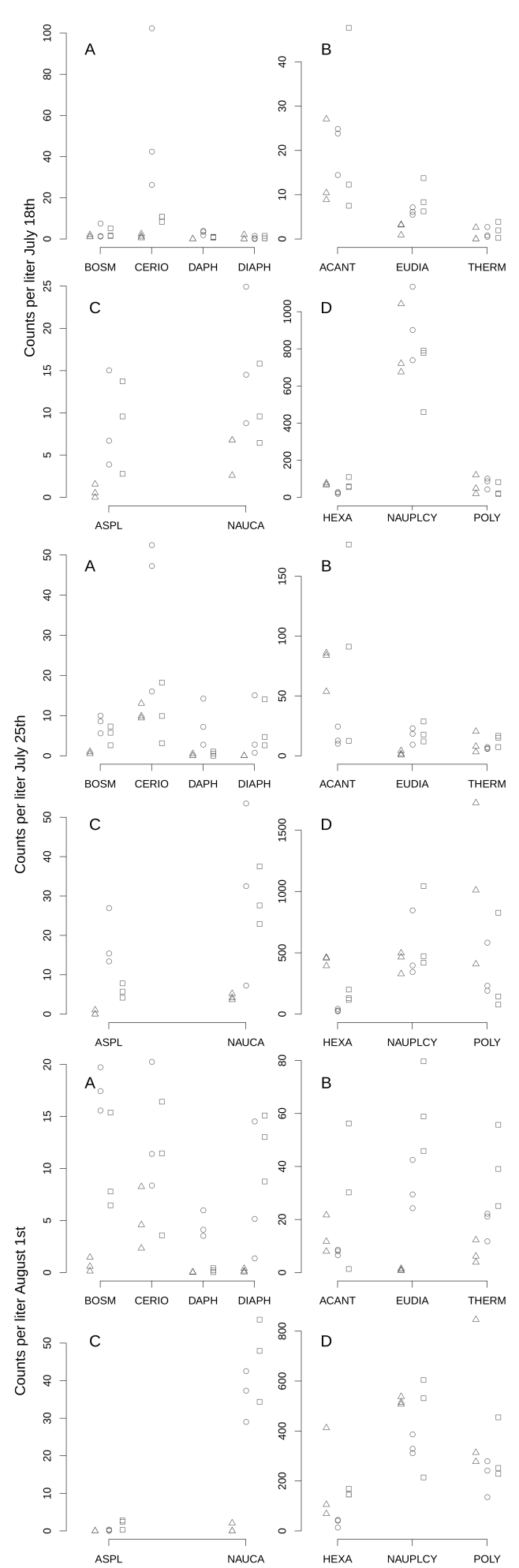

Fig. S1. Zooplankton numerical response to fish treatments at each sampling date. Circles: fishless control treatment; triangles: perch treatment, squares: roach treatment. Model predictions are not shown due to model over-parametrization resulting in inaccurate predictions. Zooplankton taxa were separated among A: Cladocerans $(\mathrm{BOSM}=$ Bosmina longirostris, $\mathrm{CERIO}=$ Ceriodaphnia pulchella and C. quadrangula, DAPH $=$ Daphnia longispina complex, DIAPH = Diaphanosoma brachyurum), B: Copepodite stages $(\mathrm{ACANT}=$ Acanthocyclops robustus, EUDIA: Eudiaptomus gracilis, THERM = Thermocyclops crassus and T. oithonoides), C: NAUCA = nauplii of Calanoids, ASPL = Asplanchna girodi and A. priodonta, D: NAUPLCY = nauplii of Cyclopoids, HEXA = Hexarthra mira, POLY $=$ Polyarthra $\mathrm{sp}$. 